\title{
Study of technical parameters of experimental traffic signs - results obtained within the frames of RID 4F research project
}

\author{
Rafał Lusa ${ }^{1,}{ }^{*}$, Pawet Skierczyński ${ }^{1}$, Leszek Kornalewski ${ }^{1}$, Adam Czyżewski ${ }^{2}$, Barbara \\ Rymsza $^{1}$, and Dariusz Litwin ${ }^{2}$ \\ ${ }^{1}$ Road and Bridge Research Institute, Instytutowa 1, 03-302 Warsaw, Poland \\ ${ }^{2}$ Maksymilian Pluta Institute of Applied Optics-INOS, Kamionkowska 18, 03-805 Warsaw, Poland
}

\begin{abstract}
This article presents the principles of selection of colours and the thickness of lines during designing of traffic signs, the legal aspects traffic signs must correspond to, test methods used during assessment of both roadway markings and vertical experimental traffic signs, results of studies of those signs and their assessment in the case of possible changes in drivers behaviour.
\end{abstract}

\section{Introduction}

The article discusses the methods for testing technical parameters of experimental signs, as well as the results of those studies. Study of technical parameters are just one of the many elements evaluated within the frames of RID $4 \mathrm{~F}$ research project - Experimental traffic signs in the aspect of behaviour of road users. Confirmation of conformity of the assessed signs with the applicable technical requirements is necessary before proceeding with evaluation of behaviour of drivers. 20 research polygons were selected for study, on which 6 different types of experimental traffic signs were located. The selected signs may be assigned to two standard types - vertical road traffic signs and roadway markings. Experimental traffic signs include anything which is not specified in the regulation on traffic signs and control devices [1]. In the future, however, it should be expected that experimental traffic signs may be manufactured from innovative materials or using innovative technology, which makes the standard test methods and the requirements inappropriate. The scope of studies of technical parameters must be determined individually for each type of marking.

\section{Objectives}

The object of the RID-4F project is to develop the guidelines specifying the activities necessary in implementation of the experimental traffic signs. Many types of studies may be used for assessment of the experimental traffic signs, their transparency and effectiveness, e.g. surveys, oculographic studies, simulations, studies of technical parameters and studies

\footnotetext{
*Corresponding author: rlusa@ibdim.edu.pl
} 
of behaviour of drivers under actual conditions. As one can see, studies of technical parameters are just one of the elements for assessment of the experimental signs. Nevertheless, it is very important that the technical parameters of the signs met the requirements over the entire period of use. Measurements of technical parameters should be carried out before acceptance of the signs and in similar periods in which the behaviours of drivers are assessed. Behaviours of drivers may be also influenced by a significant change in the level of the assessed technical parameters. Invariability of technical parameters over the entire period of use will allow us to assume that further studies and analysis are not flawed.

The article also discusses the legal acts determining the possibility of or the necessity of defining the technical requirements, the elements affecting perception of drivers, and methods of improving them.

\section{Methods}

\subsection{Guidelines for the design of experimental traffic signs}

Application of new experimental traffic signs carries some potential danger of lack of understanding of the transmitted messages by the drivers. The new signs may contain previously not used symbols, pictograms and different colour scheme than the current one. This effect can be particularly noticeable in improper design of experimental traffic signs. Although it appears that the design for new experimental traffic signs dedicated to a particular road events is a typically technical issue, certain parameters (limit values) should result from the knowledge and the limits of the physiology of the human eye, psychological knowledge about behaviours in specific situations, reception of external stimuli and assimilation of information, i.e. perception. The basic elements determining the perception of humans are: memory, attention and experience. Because the experimental traffic signs are not commonly used, recognition of that type of marking is very low. Therefore, at the design stage, it is necessary to put emphasis on the other two aspects, with particular consideration of attention. Most of the drivers are familiar with traffic signs and often even a small section is sufficient to understand the message they provide to road users. The situation is similar in the case of a time that a driver may spend on the observation of such a sign. Very often, while having a catalogue of traffic signs in memory, a "glance" is enough for the human brain to assign the sign to the transmitted messages. A completely different situation occurs with experimental traffic signs. Due to little experience, drivers do not have the new signs in memory and, what is more, they must read carefully the whole sign and understand its messages. This requires far much more time and attention of the driver, than in the case of conventional marking. These restrictions impose on experimental traffic signs much more rigorous parameters associated with the minimum and maximum size of symbols (pictograms), the font used on its face and the spacing between items on the sign, including between individual pictograms. What is more, all the elements of the sign should be visible from the same distance so that the driver, when approaching the sign, was not surprised by its new portions, which they were not able to see from a distance, and that can affect the readability of and the understanding of the contained information. 


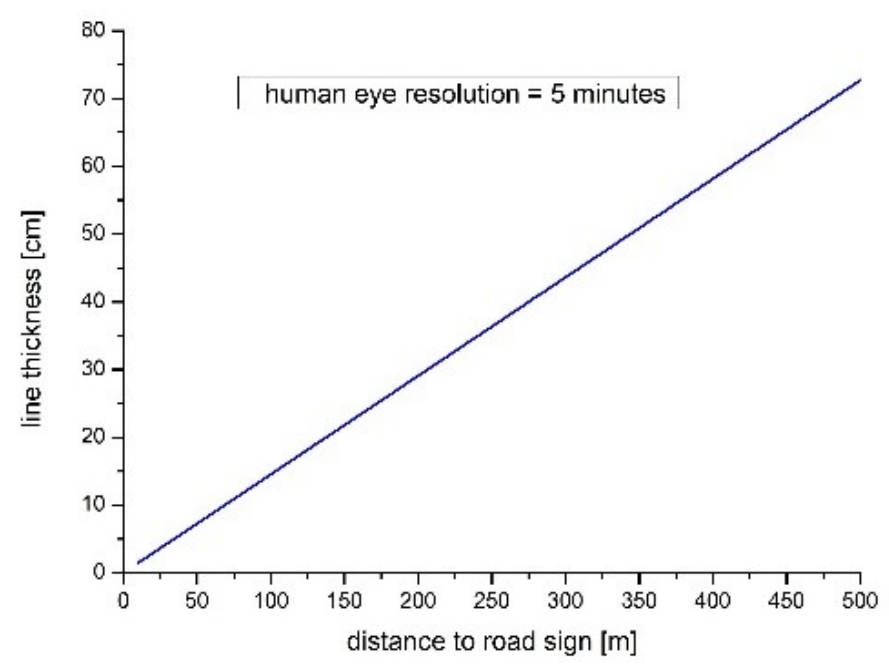

Fig. 1. Diagram of line width as a function of distance from which it is well seen by the human eye.

A single experimental traffic sign shall also not contain too much information, because this increases the observation time required to understand the transmitted messages. Hence, the dimensions of the signs should be bigger (or with maximum dimensions) from conventional marking, so that they were visible from greater distances, and thus to increase the time in which the driver will have the opportunity to review and understand the information provided on the signs. This is particularly important on motorways and express roads, where drivers can move at high speeds.

A separate parameter affecting the readability of a traffic sign is its colours and the vicinity of the colours used, which affects the contrast of the individual elements of the sign. An elementary assumption should be the use of complementary colours in the immediate vicinity and the use of light symbols (letters) on a darker background. The background of the sign should also not reflect more light than the symbol placed on it. Colours should also be similar to colours of conventional marking, which can make it easier to distinguish for example the information signs from warning signs.

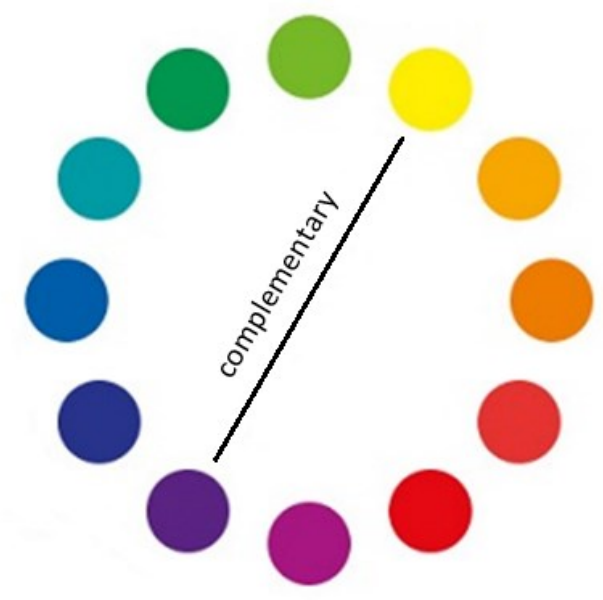

Fig. 2. Selection of contrasting colours. 


\subsection{Technical parameters}

The most important functional property of the signs, both vertical and pavement markings, is their visibility during the day, at night and in difficult weather conditions. Technical parameters specifying visibility during the day and at night will depend on the type of marking. In the case of vertical signs, the standard parameters determining visibility are:

- luminance factor $\beta$ and chromaticity coordinates $\mathrm{x}, \mathrm{y}$ (visibility during a day)

- coefficient of retroreflection RA (visibility at night)

In the case of pavement markings, the standard parameters determining visibility during the day and at night include:

- luminance coefficient in diffused light QD or luminance factor $\beta$ (visibility during the day)

- coefficient of retroreflection RL (visibility at night)

- chromaticity coordinates $\mathrm{x}, \mathrm{y}$ (visibility during the day)

- skid resistance SRT

Measurements of luminance factor $\beta$, luminance coefficient in diffused light QD, coefficient of retroreflection RL and the skid resistance SRT were conducted in accordance with the methods described in PN-EN 1436+A1:2008 [2].

Measurements of luminance factor $\beta$ and chromaticity coordinates $\mathrm{x}, \mathrm{y}$ and the coefficient of retroreflection RA were conducted in accordance with the methods described in PN-EN 12899-1:2010 [3].

The coefficient of retroreflection RA is defined as the light intensity coefficient $\mathrm{R}$ in relation to the specific surface A. The coefficient of retroreflection RA is a measure of efficiency of reflection of the specific surface.

$$
R_{A}=\frac{R}{A}=\frac{I}{E_{i} \cdot A}
$$

where:

$R_{A}$ - coefficient of retroreflection $[\mathrm{cd} \cdot \mathrm{m}-2 \cdot \mathrm{lx}-1]$

$R$ - light intensity coefficient [cd $\cdot 1 \mathrm{x}-1]$

$E_{i}$ - illumination in location of retroreflector in a plane perpendicular to the axis of lighting [lux]

$I$ - Luminous intensity [cd]

$A-$ area [m2]

The new study proposed in the RID-4F project was the measurement of uniplanarity of large road boards. The uniplanarity of road boards has impact on uniform perception of a road board at night. If the board is properly manufactured, the entrance angle $\theta 2$ and the observation angle $\theta 1$ are identical for the entire sign. When failing to meet the uniplanarity requirement, those angles change depending on the sign area, which affects the change of luminance of the sign. As a result, we will observe darker and lighter fields.

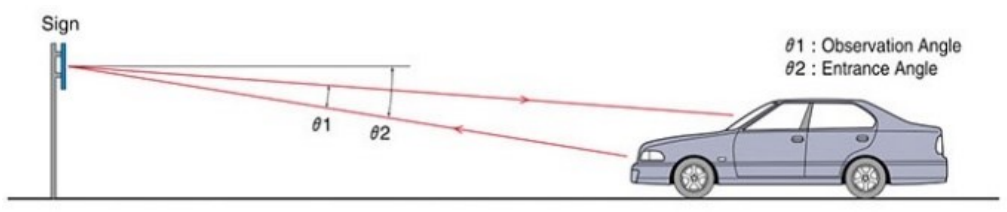

Fig. 3. Diagram presenting reflection of headlights from a traffic sign and return to the driver's eyes, taking into account the angle of observation and the angle of illumination. Source: http://reflectivetape.info/wp-content/uploads/2011/05/ulg_reflect.jpg. 
For the measurement of uniplanarity, Topcon DS-203i scanning tachymeter was used. The tachymeter scans the selected surface at a set frequency. As a result we obtain a grid of points with coordinates $\mathrm{x}, \mathrm{y}, \mathrm{z}$. Based on the received grid of points, the most matched plane was assigned in the CloudCompare software and deviations were calculated on that basis. The most matched plane a theoretical, perfectly flat plane of the sign.

\section{Results}

Test results should be the basis for developing guidelines for the selection of technical parameters of the experimental traffic signs, test methods and requirements and method of assessment. The currently functioning marking may be divided due to location of placing in the right-of-way (vertical signs and pavement markings) or due to the method of transmitting the messages (reflecting or emitting light). In the future we may have to deal with new signs transmitting messages, e.g. using sounds, holograms, displaying symbols on the right-of-way or using the connection of all the types. The only limitation is human imagination. Therefore, it is not possible to determine in advance the requirements for all experimental traffic signs.

Under the current legislation there are no requirements for experimental traffic signs; therefore, it is assumed that they must meet the requirements for standard markings. In the RID-4F study, all experimental traffic signs had the features of standard markings, therefore the requirements were assumed in accordance with the currently applicable legal acts. Requirements for standard traffic signs are specified in the Regulation [4] and the standards [2] and [3].

Due to the large number of tested types of signs (more than 20), it was decided to present the results only for selected types. For vertical signs, the results for the sign directing to the VIATOLL gates on the A1 motorway downstream the fee collection point in Stryków (Fig. 4).

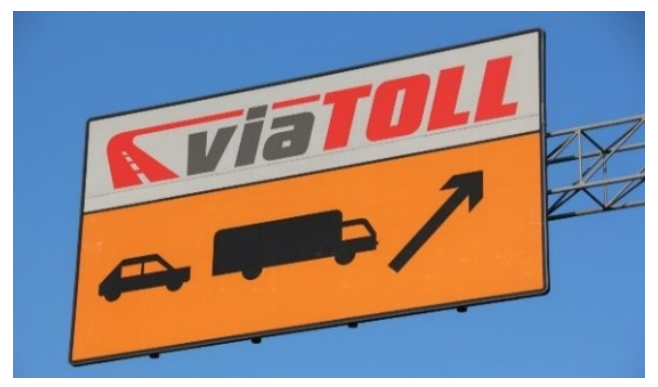

Fig. 4. Vertical signs directing to VIATOLL gates.

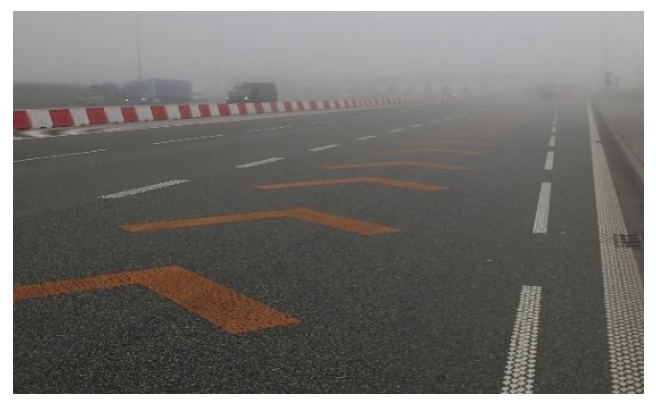

Fig. 5. Road markings directing to VIATOLL gates. 
Also pavement markings directing to the VIATOLL gates (so-called "guards"), were also located in the same place and will be representative for studies of pavement markings (Fig. 5). The results of study of vertical signs are presented in Table 1, Table 2 and Table 3.

The results of study of pavement markings are presented in Table 4. Fig. 7. presents the results of flatness tests (uniplanarity) of the vertical sign. The sign is scanned using a scanning tachymeter with a random interval of distance between the points. It is recommended that the distance between the points was no greater than $20 \mathrm{~cm}$. This results in obtaining a grid of points depicting the "flatness" of the sign board. Deviations are calculated from the best matched plane designated based on that grid of points. Because it is a new study, the requirements for a maximum value of deviations has not been yet developed.

Table 1. Results of coefficient of retroreflection $R_{A}$ of a VIATOLL vertical traffic sign.

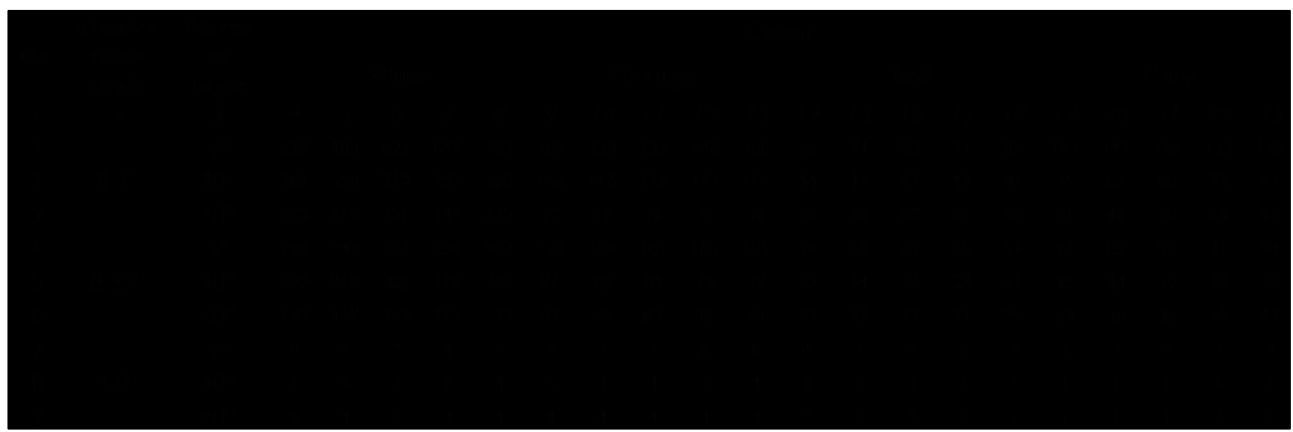

Table 2. Average results of coefficient of retroreflection $R_{A}$ of a VIATOLL vertical traffic sign.

\begin{tabular}{|c|c|c|c|c|c|c|}
\hline \multirow{2}{*}{ No. } & \multirow{2}{*}{ Observation angle } & \multirow{2}{*}{ Entrance angle } & \multicolumn{4}{|c|}{ Colour } \\
\cline { 3 - 7 } & & & White & Orange & Red & Grey \\
\hline 1 & 2 & 3 & 4 & 5 & 6 & 7 \\
\hline 1 & \multirow{2}{*}{$0.2^{\circ}$} & $5^{\circ}$ & 569 & 514 & 465 & 388 \\
2 & & $30^{\circ}$ & 335 & 285 & 237 & 198 \\
3 & & $40^{\circ}$ & 211 & 180 & 152 & 120 \\
\hline 4 & \multirow{2}{*}{$0.33^{\circ}$} & $5^{\circ}$ & 273 & 260 & 243 & 218 \\
5 & & $30^{\circ}$ & 214 & 184 & 156 & 131 \\
6 & & $40^{\circ}$ & 160 & 138 & 117 & 94 \\
\hline 7 & \multirow{2}{*}{$2.0^{\circ}$} & $5^{\circ}$ & 5 & 5 & 5 & 6 \\
8 & & $30^{\circ}$ & 3 & 4 & 4 & 4 \\
9 & & $40^{\circ}$ & 4 & 4 & 4 & 4 \\
\hline
\end{tabular}




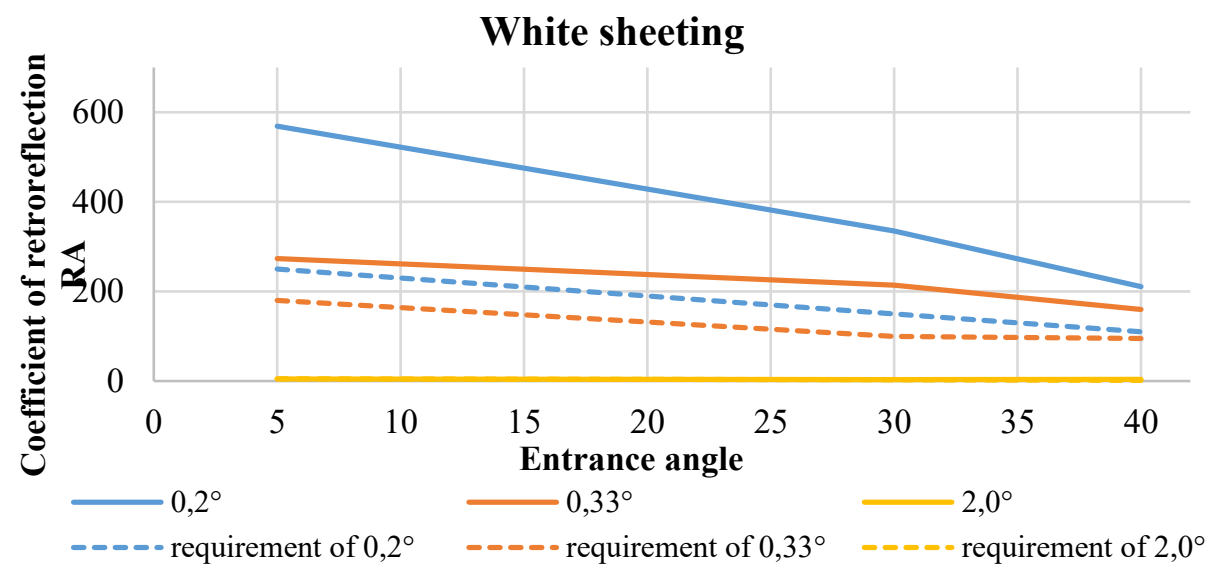

Fig. 6. Coefficient of retroreflection $R_{A}$ of a VIATOLL vertical traffic sign together with a requirement.

Table 3. Average results of luminance factor $\beta$ and chromaticity coordinates $x, y$ of a VIATOLL vertical traffic sign.

\begin{tabular}{|c|c|c|c|c|}
\hline No. & Sign type & Colour & Luminance factor $\beta$ & Chromaticity coordinates $x, y$ \\
\hline 1 & 2 & 3 & 4 & 5 \\
\hline 1 & \multirow{10}{*}{ VIATOLL } & \multirow{2}{*}{ White } & \multirow{2}{*}{0.301} & 0.316 \\
\hline 2 & & & & 0.333 \\
\hline 3 & & \multirow{2}{*}{ Orange } & \multirow{2}{*}{0.224} & 0.525 \\
\hline 4 & & & & 0.420 \\
\hline 5 & & \multirow{2}{*}{ Red } & \multirow{2}{*}{0.120} & 0.586 \\
\hline 6 & & & & 0.327 \\
\hline 7 & & \multirow{2}{*}{ Grey } & \multirow{2}{*}{0.075} & 0.321 \\
\hline 8 & & & & 0.337 \\
\hline 9 & & \multirow{2}{*}{ Black } & \multirow{2}{*}{0.012} & 0.303 \\
\hline 10 & & & & 0.321 \\
\hline
\end{tabular}

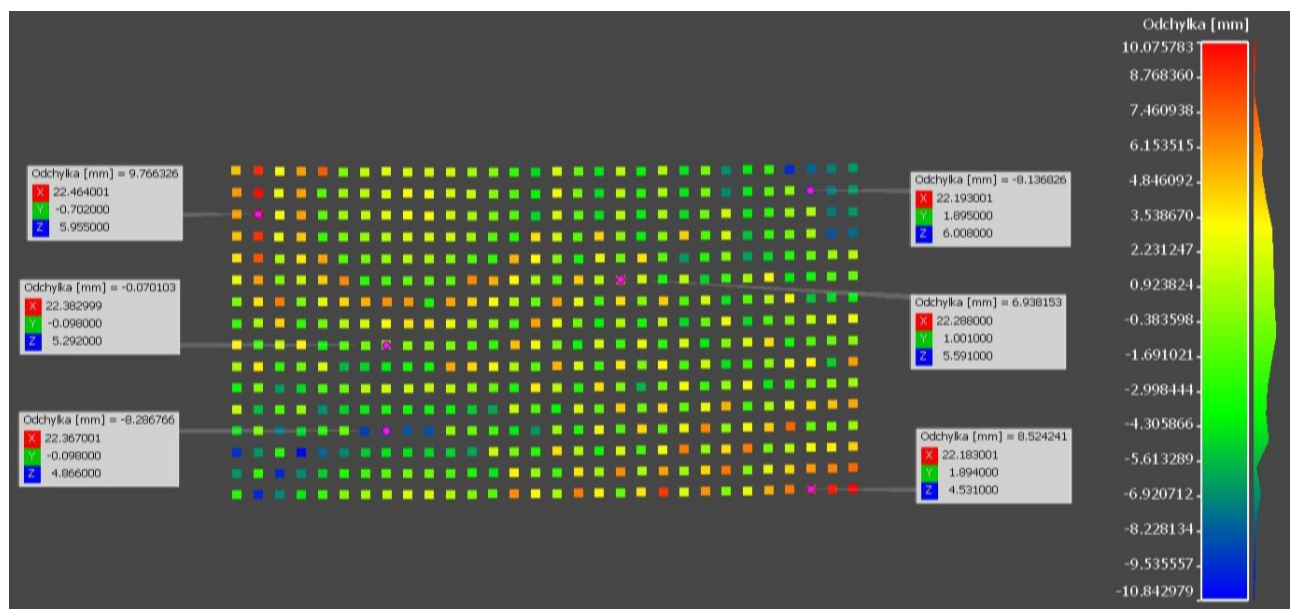

Fig. 7. Grid of points depicting flatness of a VIATOLL vertical sign (deviations from the best matched plane). 
Table 4. Results of tests of road markings directing to VIATOLL gates.

\begin{tabular}{|c|c|c|c|c|c|c|c|}
\hline No. & Property & \multicolumn{5}{|c|}{ Test results } & Average \\
\hline 1 & 2 & 3 & 4 & 5 & 6 & 7 & 8 \\
\hline 1 & Coefficient of retroreflection $\mathrm{R}_{\mathrm{L}}$ & 21 & 23 & 25 & 25 & 25 & 24 \\
\hline 2 & Luminance coefficient in diffused light QD & 113 & 101 & 95 & 109 & 106 & 105 \\
\hline 3 & Skid resistance SRT & 53 & 53 & 52 & 52 & 52 & 52 \\
\hline 4 & Luminance factor $\beta$ & 0.135 & 0.141 & 0.155 & 0.138 & 0.142 & 0.142 \\
\hline 5 & 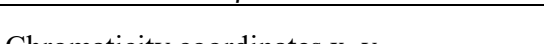 & 0.435 & 0.446 & 0.442 & 0.441 & 0.438 & 0.440 \\
\hline 6 & Chromaticity coordinates $\mathrm{x}, \mathrm{y}$ & 0.392 & 0.398 & 0.393 & 0.395 & 0.393 & 0.394 \\
\hline
\end{tabular}

\section{Conclusions}

When evaluating the results of technical tests, the requirements for the markings at the stage of designing must be taken into account. Because we are dealing with experimental traffic signs, it should first of all meet the requirements of the technical specification. In the case of colours, changes are permitted only in justified cases. When it comes to the evaluation of the experimental vertical signs, attention must be paid to the "exit" of chromaticity coordinates $\mathrm{x}, \mathrm{y}$ of the orange colour out of its colour field. The other examined parameters such as coefficient of retroreflection $\mathrm{R}_{\mathrm{A}}$, luminance factor $\beta$ and the remaining colours meet the requirements of the standard. In the case of pavement markings, attention must be paid to lack of reflection of the assessed marking. Because marking is located in the illuminated area of the toll collection point, failure to meet the requirement of reflection is permitted. However, it is necessary to analyse whether the loss of reflection could have an impact on the behaviour of drivers. The colour of arrows directing to the VIATOLL gates also does not fit in the orange colour range.

The most important reason for technical tests of experimental traffic sign is the full awareness of changes of parameters of those signs in time and the evaluation of impact of those changes on behaviour of the driver. It should be noted that all the applicable traffic signs should guarantee good visibility during the day and at night throughout the period of use, which has a direct impact on the safety of the drivers.

\section{References}

1. The Regulation of the Minister of Infrastructure and the Minister of the Interior and Administration of 31 July 2002 on traffic signs and control devices (Journal of Laws No. 170, item 1393, as amended) (Poland, 2002)

2. PN-EN 1436:2007+A1:2008 Road marking materials. Road marking performance for road users (2008)

3. PN-EN 12899-1:2010 Fixed vertical road traffic signs. Part 1: Fixed road signs (Poland, 2010)

4. Regulation of the Minister of Infrastructure of 3 July 2003r. on detailed technical conditions for road signs and signals and traffic safety devices and on the conditions for their location on the road, OJ 2003 No. 220 Pos. 2181 as amended (Poland, 2003)

5. CIE 15:2004 Technical Report: Colorimetry, 3rd edition (International Commission on Illumination, 2004)

6. CIE 54.2:2001 Retroreflection: Definition And Measurement (International Commission on Illumination, 2001) 